\title{
COURRIER
}

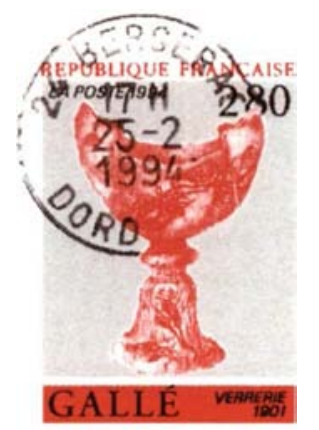

\section{Bioéthique et diagnostic prénatal}

Dans son article "Bioéthique et Démocratie " $\left(\mathrm{m} / \mathrm{s} n^{\circ}\right.$ 6-7, vol. 10, p. 750), Bernard Calvino reprend l’idée couramment admise que la pratique du diagnostic prénatal peut être la source d'un "eugénisme thérapeutique ", si elle se généralise à toutes les maladies à transmission génétique. L'auteur précise que l'eugénisme consiste en "l'élimination des mauvais gènes".

Lorsque le diagnostic prénatal est appliqué à des maladies à transmission génétique comme des translocations équilibrées ou des maladies récessives autosomiques ou liées à l'X, il aboutit, certes, à l'élimination de sujets génétiquement atteints, mais il permet, en revanche, la naissance d'individus normaux qui, pour un très grand nombre d'entre eux, n'auraient pas vu le jour et dont $50 \%$ sont des vecteurs de ces "mauvais gènes " que l'eugénisme se propose d'éliminer. Loin d'être une pratique eugénique, le diagnostic prénatal se révèle, au contraire, néfaste pour le patrimoine génétique de l'humanité.

Pour éradiquer une maladie gémétique, il faudrait éliminer tous les foetus qui se révèlent être des vecteurs sains. Ce ne pourrait être qu’une décision "politique" que seul un régime dictatorial serait en mesure de faire appliquer. Les résultats seraient bien décevants, car si ces "mauvais gènes" sont nombreux, ils sont, pris individuelle$\mathrm{m} / \mathrm{s} n^{\circ} 10$, vol. 10 , octobre 94 ment, extrêmement rares et de ce fait leur élimination est pratiquement irréalisable. De plus, l'apparition de mutations échapperait à une telle pratique.

En réalité, le diagnostic prénatal intéresse très peu de couples porteurs de ces "mauvais gènes", mais surtout des sujets sans antécédents génétiques particuliers, car ce sont les mutations chromosomiques qui sont recherchées. Dans ce cas, les enfants qui naîtront seront, en principe, tous "génétiquement normaux " contrairement au cas des maladies à transmission génétique. Enfin, l'élimination du foetus atteint d'anomalie chromosomique n'entre pas dans le cadre d'une démarche eugénique qui a pour objet d'éliminer les anomalies susceptibles d'être transmises, car il n'y a ici pratiquement aucun risque de propagation de l'anomalie dans la population.

Le diagnostic prénatal est une démarche médicale qui n'a rien à voir avec l'eugénisme, notion essentiellement politique et dénuée de tout fondement scientifique

\section{François Picard}

Institut de cytogénétique, Faculté de médecine, avenue du Professeur LéonBernard, 35043 Rennes Cedex, France. 